\title{
FUNCTIONS POSSESSING RESTRICTED MEAN VALUE PROPERTIES ${ }^{1}$
}

\author{
DAVID HEATH
}

\begin{abstract}
A real-valued function $f$ defined on an open subset of $\boldsymbol{R}^{N}$ is said to have the restricted mean value property with respect to balls (spheres) if, for each point $x$ in the set, there exists a ball (sphere) with center $x$ and radius $r(x)$ such that the average value of $f$ over the ball (sphere) is equal to $f(x)$. If $f$ is harmonic then it has the restricted mean value property. Here new conditions for the converse implication are given.
\end{abstract}

1. Introduction. Suppose $D \subseteq R^{N}$ is open and $r: D \rightarrow R$ is positive and $r(x) \leqq d\left(x, D^{c}\right)$, the distance from $x$ to the complement of $D$. Let $B(x, r)$ denote the open ball of radius $r$ about $x$, and $S(x, r)$ the corresponding sphere. If $f: D \rightarrow R$ satisfies

$$
f(x)=\frac{1}{\mu(B(x, r(x)))} \int_{B(x, r(x))} f(y) \mu(d y), \quad \forall x \in D,
$$

where $\mu$ is Lebesgue measure in $\boldsymbol{R}^{N}$, we say that $f$ possesses the restricted mean value property on balls (in $D$ with respect to $r$ ). If $f$ satisfies

$$
f(x)=\frac{1}{v(S(x, r(x)))} \int_{S(x, r(x))} f(y) \nu(d y), \quad \forall x \in D,
$$

where $v(d y)$ is the element of surface area on $S(x, r(x))$, we say that $f$ possesses the restricted mean value property on spheres. Of course every harmonic $f$ possesses the restricted mean value property on balls and spheres for arbitrary $r$; we are concerned here with partial converses to this fact.

Several partial converses are known: Courant and Hilbert [4] prove that if $D$ is relatively compact and regular for the Dirichlet problem and $f$ is continuous on $\bar{D}$ and possesses the restricted mean value property on spheres, then $f$ is harmonic. Föllmer [6], using probabilistic techniques, proved a similar result (which he also generalized) requiring $r$ to be Borel measurable, but his only assumption on $D$ is that Brownian paths leave $D$

Received by the editors February 5, 1973.

AMS (MOS) subject classifications (1970). Primary 60J45, 31B05.

Key words and phrases. Harmonic function, mean value property, Markov process.

${ }^{1}$ Research supported in part by the National Science Foundation.

(c) American Mathematical Society 1973 
almost surely and $f$ need be continuous only almost everywhere on $\partial D$ and on all of $D$.

Baxter [2], extending a result of Ackoglu and Sharpe [1], showed that if $\bar{D}$ is a compact $C^{1}$ manifold with boundary and $r$ is measurable and satisfies $r(x) \geqq \varepsilon_{0} d\left(x, D^{c}\right)$, then any bounded measurable $f$ possessing the restricted mean value property on balls is harmonic.

More recently, Veech [7] and [8] showed that if $N=2$, and in [9], if $N \geqq 1, D$ is a relatively compact Lipschitz domain, and $r$ is bounded away from zero on compact subsets of $D$, then every Lebesgue measurable function whose absolute value is dominated by some harmonic function on $D$ and which possesses the restricted mean value property on balls is harmonic.

In this paper we shall prove the following two theorems:

THEOREM 1. Suppose $f$ is bounded, $D$ is a proper subset of $\boldsymbol{R}^{N}$ and $r$ satisfies

$$
\varepsilon d\left(x, D^{c}\right)<r(x)<(1-\varepsilon) d\left(x, D^{c}\right)
$$

for some $\varepsilon>0$. Iff possesses the restricted mean value property on balls, then $f$ is harmonic.

THEOREM 2. Suppose $f$ is bounded, $N \geqq 2$, and $r$ satisfies

$$
|r(x)-r(y)|<\left(1-\varepsilon_{0}\right)|x-y|
$$

for some $\varepsilon_{0}>0$. If $f$ possesses the restricted mean value property on spheres then $f$ is harmonic.

2. Preliminary results. Suppose $\left(\Omega^{0}, x^{0}, \mathscr{F}_{t}^{0}, \theta_{t}^{0}, P_{x}^{0}, x \in R^{N}\right)$ is standard $N$-dimensional Brownian motion. Suppose $\left(\rho_{n}^{1}\right)$ is a sequence of independent, identically distributed random variables on $\left(\Omega^{1}, \mathscr{F} 1, P^{1}\right)$ with distribution function $G$ defined by

$$
\begin{array}{rlrl}
G(x)=0, & & \text { if } x \leqq 0, \\
& =x^{N}, & & \text { if } 0 \leqq x \leqq 1, \\
& =1, & & \text { if } 1 \leqq x .
\end{array}
$$

Let $\Omega=\Omega^{0} \times \Omega^{1}, P_{x}=P_{x}^{0} \times P^{1}, \mathscr{F}_{t}=\mathscr{F}_{t}^{0} \times \mathscr{F}^{1}$, and define $\theta_{t}$ by

$$
\theta_{t}\left(\omega^{0}, \omega^{1}\right)=\left(\theta_{t}^{0}\left(\omega^{0}\right), \omega^{1}\right) \text {. }
$$

Extend $x^{0}$ and $\rho_{n}^{1}$ by $x\left(t,\left(\omega^{0}, \omega^{1}\right)\right)=x^{0}\left(t, \omega^{0}\right)$ and $\rho_{n}\left(\omega^{0}, \omega^{1}\right)=\rho_{n}^{1}\left(\omega^{1}\right)$. Given $r: D \rightarrow R$, measurable, with $0<r(x)<d\left(x, D^{c}\right)$ we define two sequences of stopping times as follows:

$$
\begin{aligned}
\tau_{0}(\omega) & =0, \\
\tau_{n+1}(\omega) & =\inf \left\{t: t \geqq \tau_{n}(\omega),\left|x(t, \omega)-x\left(\tau_{n}(\omega), \omega\right)\right| \geqq \rho_{n+1}(\omega) \cdot r\left(x\left(\tau_{n}(\omega), \omega\right)\right)\right\}
\end{aligned}
$$


and

$$
\begin{aligned}
\sigma_{0}(\omega) & =0, \\
\sigma_{n+1}(\omega) & =\inf \left\{t: t \geqq \sigma_{n}(\omega),\left|x(t, \omega)-x\left(\sigma_{n}(\omega), \omega\right)\right| \geqq r\left(x\left(\sigma_{n}(\omega), \omega\right)\right)\right\} .
\end{aligned}
$$

Let $\mathscr{G}_{n}$ and $\mathscr{H}_{n}$ denote the minimal $\sigma$-fields for the sequences $x\left(\tau_{n}\right)$ and $x\left(\sigma_{n}\right)$ respectively. Then clearly $\left(x\left(\tau_{n}\right), \mathscr{G}_{n}\right)$ is a Markov chain and the conditional distribution of $x\left(\tau_{n+1}\right)$ given $x\left(\tau_{n}\right)$ is uniform on $B\left(x\left(\tau_{n}\right), r\left(x\left(\tau_{n}\right)\right)\right)$; a similar statement holds for $x\left(\sigma_{n+1}\right)$ and $S\left(x\left(\sigma_{n}\right), r\left(x\left(\sigma_{n}\right)\right)\right)$.

Define $\tau$ by $\tau(\omega)=\inf \{t \geqq 0: x(t, \omega) \notin D\}$ if this set is nonempty; otherwise set $\tau(\omega)=+\infty$.

LEMma 1. If $r$ satisfies (1.3) then $\lim _{n \rightarrow \infty} \tau_{n}(\omega)=\tau(\omega) P_{x}$-a.s. for every $x \in D$. If $r$ satisfies (1.4) then $\lim _{n \rightarrow \infty} \sigma_{n}(\omega)=\tau(\omega) P_{x}$-a.s. for every $x \in D$.

Proof. We prove only the first statement: Let $\tau^{\prime}(\omega)=\lim _{n \rightarrow \infty} \tau_{n}(\omega)$; clearly $\tau^{\prime} \leqq \tau$ since $\tau_{n} \nearrow$ and $\tau_{n} \leqq \tau$. Suppose $\tau^{\prime}(\omega)<\infty$. Then $x\left(\tau^{\prime}(\omega), \omega\right)=$ $\lim _{n \rightarrow \infty} x\left(\tau_{n}(\omega), \omega\right)$. If $x\left(\tau^{\prime}(\omega), \omega\right) \in D$ then $d\left(x\left(\tau^{\prime}(\omega), \omega\right), D^{c}\right)>0$, so by (1.3),

$$
\underset{n \rightarrow \infty}{\liminf } r\left(x\left(\tau_{n}(\omega), \omega\right)\right)>0
$$

But then

$$
\underset{n \rightarrow \infty}{\limsup } \rho_{n+1}(\omega)=\limsup _{n \rightarrow \infty} \frac{\left|x\left(\tau_{n+1}(\omega), \omega\right)-x\left(\tau_{n}(\omega), \omega\right)\right|}{r\left(x\left(\tau_{n}(\omega), \omega\right)\right)}=0 ;
$$

this event has probability zero so $\tau^{\prime}(\omega)<\infty \Rightarrow x\left(\tau^{\prime}(\omega), \omega\right) \notin D \Rightarrow \tau(\omega) \leqq$ $\tau^{\prime}(\omega)$.

REMARK. We clearly have

$$
\begin{aligned}
\bigcup_{i=m}^{\infty} B\left(x\left(\tau_{i}(\omega), \omega\right),\right. & \left.r\left(x\left(\tau_{i}(\omega), \omega\right)\right)\right) \\
\supseteq & \left\{y: y=x(t, \omega) \text { for some } t \in\left[\tau_{i}(\omega), \tau(\omega)\right)\right\} .
\end{aligned}
$$

This inclusion remains valid if all $\tau_{i}$ 's are replaced by $\sigma_{i}$ 's.

Lemma 2. Suppose $f$ is bounded and measurable on $D$ and satisfies (1.1) or (1.2). There is a bounded measurable function $F$ on $\Omega$ such that $f(x)=$ $E_{x}(F(\omega)) \forall x \in D$.

Proof. If $f$ satisfies (1.1) then $f\left(x\left(\tau_{n}(\omega), \omega\right)\right)$ is a bounded martingale, so by the martingale convergence theorem it converges to some function $F P_{x}$-a.s. for every $x \in D$ and the above equation holds. If $f$ satisfies (1.2), replace $\tau_{n}$ by $\sigma_{n}$ in the above argument. 
For a proof of the following result, see Chung [3]:

Lemma 3. Suppose $(M, \mathfrak{A}, m)$ is a probability space and $\left(\mathfrak{H}_{n}\right)$ is an increasing sequence of $\sigma$-fields with $\mathfrak{A}_{n} \subseteq \mathfrak{U}$. Let $\mathfrak{H}_{\infty}=\bigvee_{\mathfrak{1}}^{\infty} \mathfrak{A}_{n}$, and suppose $A_{i} \in \mathfrak{U}_{\infty}$. Then

$$
\lim _{n \rightarrow \infty} P\left[\bigcap_{i=n}^{\infty} A_{i} \mid \mathfrak{A}_{n}\right]=1_{\cup_{m=1}^{\infty}} \cap_{i=m}^{\infty} A_{i} \cdot
$$

Lemma 4. Under the hypotheses of Theorem 2, there exists a $\delta>0$ such that if $x_{i}, A_{i}, i=1,2$, satisfy $x_{i} \in D, A_{i}$ measurable and $A_{i} \subseteq D, P^{2}\left(x_{i}, A_{i}\right)>$ $1-\delta$ and $x_{2} \in B\left(x_{1}, r\left(x_{1}\right)\right)$ then $A_{1} \cap A_{2} \neq \varnothing$.

Proof. Define $A\left(x, r_{1}, r_{2}\right)=\left\{y: r_{1}<|y-x|<r_{2}\right\}$, let $\varepsilon^{\prime}=\varepsilon_{0} / 4$ and let $x$ denote either $x_{1}$ or $x_{2}$. Suppose $y \in A\left(x,\left(1-\varepsilon^{\prime}\right) r(x),\left(1+\varepsilon^{\prime}\right) r(x)\right)$ and $a$ is chosen small enough that $B(y, a) \subseteq A\left(x,\left(1-\varepsilon^{\prime}\right) r(x),\left(1+\varepsilon^{\prime}\right) r(x)\right)$. We shall show that

$$
P^{2}(x, B(y, a)) \geqq c\left(\varepsilon_{0}\right) a^{N} /(r(x))^{N} .
$$

To this end, let $C=\{z \in S(x, r(x)):|r(z)-| z-y|| \leqq a / 2\}$. (Note that $|r(z)-| z-y||$ is the distance by which the sphere $S(z, r(z))$ misses the point $y$.) Simple estimates of the central angle of the part of the sphere $S(z, r(z))$ which is contained in $B(y, a)$ give

$$
z \in C \Rightarrow P^{1}(z, B(y, a)) \geqq c_{1}\left(\varepsilon_{0}\right) a^{N-1} /(r(x))^{N-1} .
$$

Using the inequalities on (and also the continuity of) $r$, one shows easily that $x+r(x)((y-x) /|y-x|)$ (respectively $x-r(x)((y-x) /|y-x|))$ are not in $C$ because $r$ is too big (small) there, and hence the intersection of $C$ with each great circle on $S(x, r(x))$ through $x+r(x)((y-x) /|y-x|)$ is nonvoid; moreover, the measure of this intersection and the distance of the intersection from $x \pm r(x)((y-x) /|y-x|)$ are easily shown to be bounded below appropriately so that $P^{1}(x, C) \geqq c_{2}\left(\varepsilon_{0}\right) a / r(x)$. (2.1) now follows easily. Notice that (2.1) implies that for any measurable

$$
E \subseteq A\left(x,\left(1-\varepsilon^{\prime}\right) r(x),\left(1+\varepsilon^{\prime}\right) r(x)\right)
$$

we have

$$
P^{2}(x, E) \geqq c\left(\varepsilon_{0}\right) \mu(E) /(r(x))^{N} .
$$

To complete the proof, set

$$
\begin{aligned}
I= & A\left(x_{1},\left(1-\varepsilon^{\prime}\right) r\left(x_{1}\right),\left(1+\varepsilon^{\prime}\right) r\left(x_{1}\right)\right) \\
& \cap A\left(x_{2},\left(1-\varepsilon^{\prime}\right) r\left(x_{2}\right),\left(1+\varepsilon^{\prime}\right) r\left(x_{2}\right)\right) .
\end{aligned}
$$

An elementary computation (again using the properties of $r$ ), using the 
fact that $x_{2} \in B\left(x_{1}, r\left(x_{1}\right)\right)$, shows that

$$
\mu(I) \geqq k_{1}\left(\varepsilon_{0}\right) r\left(x_{1}\right)^{N-1} r\left(x_{2}\right) \geqq k\left(\varepsilon_{0}\right) r\left(x_{i}\right)^{N}, \quad i=1,2 .
$$

We now select $\delta$ so that under the hypotheses of the theorem we can conclude $\mu\left(I \cap A_{1}^{c}\right)+\mu\left(I \cap A_{2}^{c}\right)<\mu(I)$ so that $A_{1} \cap A_{2}$ must be nonvoid.

From (2.2) we obtain $\mu\left(I \cap A_{i}^{c}\right) \leqq\left(r\left(x_{i}\right)\right)^{N} P^{2}\left(x_{i}, I \cap A_{i}^{c}\right) / c\left(\varepsilon_{0}\right)$, so that by (2.3)

$$
\mu\left(I \cap A_{i}^{c}\right) / \mu(I) \leqq P^{2}\left(x_{i}, I \cap A_{i}^{c}\right) / c\left(\varepsilon_{0}\right) k\left(\varepsilon_{0}\right) .
$$

Hence if $\delta=c\left(\varepsilon_{0}\right) k\left(\varepsilon_{0}\right) / 4$ we obtain

$$
\mu\left(I \cap A_{1}^{c}\right)+\mu\left(I \cap A_{2}^{c}\right) \leqq \mu(I) / 2<\mu(I)
$$

as desired. This completes the proof of Lemma 4.

Lemma 5. Suppose $r$ satisfies (1.3). Let $P^{1}(x, A)$ denote the one-step transition probability for the chain $x\left(\tau_{n}(\omega), \omega\right)$. Then there is a $\delta>0$ such that if $x_{i}, A_{i}, i=1,2$, satisfy $P^{1}\left(x_{i}, A_{i}\right) \geqq 1-\delta, x_{i} \in D, A_{i} \subseteq D, x_{2} \in$ $B\left(x_{1}, r\left(x_{1}\right)\right)$, then $A_{1} \cap A_{2} \neq \varnothing$.

Proof. Apply simple geometry and use the techniques of the proof of the previous lemma.

\section{Proofs of the theorems.}

Proof of Theorem 1. By a simple modification of an elementary but clever argument of Veech, [7] or [9], we may and shall assume that $f$ and $r$ are Borel measurable. Let $x_{0} \in D$ and $0<r_{0}<d\left(x_{0}, D^{c}\right)$. It suffices to show that

$$
f\left(x_{0}\right)=\frac{1}{v\left(S\left(x_{0}, r_{0}\right)\right)} \int_{S\left(x_{0}, r_{0}\right)} f(y) v(d y) .
$$

Let $y_{n}(\omega)=x\left(\tau_{n}(\omega), \omega\right)$. By Lemma $2 \lim _{n \rightarrow \infty} f\left(y_{n}\right)=F$ exists $P_{x}$-almost surely and $f(x)=E_{x}(F)$. Let $\eta(\omega)=\inf \left\{t>0:\left|x(t, \omega)-x_{0}\right| \geqq r_{0}\right\}$ and $\theta_{\eta}$ be the corresponding shift operator. Now by the strong Markov property (see Dynkin [6, p. 100]):

$$
E_{x_{0}}\left(F \cdot \theta_{\eta} \mid \mathscr{F}_{\eta}\right)=E_{x_{\eta}}(F) \quad P_{x_{0}} \text {-a.s., } \quad x_{\eta}=x(\eta(\omega), \omega) .
$$

It therefore suffices to show that $F \cdot \theta_{\eta}=F P_{x_{0}}$-a.s., for then

$$
\begin{aligned}
f\left(x_{0}\right) & =E_{x_{0}}(F)=E_{x_{0}}\left(E_{x_{0}}\left(F \mid \mathscr{F}_{\eta}\right)\right)=E_{x_{0}}\left(E_{x_{0}}\left(F \cdot \theta_{\eta} \mid \mathscr{F}_{\eta}\right)\right) \\
& =E_{x_{0}}\left(E_{x_{\eta}}(F)\right)=E_{x_{0}}\left(f\left(x_{\eta}\right)\right)=\frac{1}{v\left(S\left(x_{0}, r_{0}\right)\right)} \int_{S\left(x_{0}, r_{0}\right)} f(y) v(d y) .
\end{aligned}
$$

Now consider the sequence $z_{n}(\omega)=y_{n}\left(\theta_{\eta}(\omega)\right)$. Clearly $f\left(z_{n}\right)$ is a martingale which converges to $F \cdot \theta_{\eta}(\omega) P_{x_{0}}$-a.s. Thus we need only show that $\lim _{n \rightarrow \infty} f\left(z_{n}(\omega)\right)=\lim _{n \rightarrow \infty} f\left(y_{n}(\omega)\right)$. 
From the definition of the process $z_{n}$, an argument similar to the proof of Lemma 1, and the remark following Lemma 1 we see that: For each $m$ there is an $n_{0}(\omega)$ such that $n \geqq n_{0}(\omega) \Rightarrow z_{n}(\omega) \in \bigcup_{i=m}^{\infty} B\left(y_{i}(\omega), r\left(y_{i}(\omega)\right)\right)$.

Suppose now that $\varepsilon>0$ and $K>0$ are prescribed. Let

$$
\begin{aligned}
A_{i}^{\varepsilon} & =\left\{\omega:\left|f\left(y_{i+1}(\omega)\right)-f\left(y_{i}(\omega)\right)\right|<\varepsilon / 2\right\}, \\
B_{i}^{\varepsilon} & =\left\{\omega:\left|f\left(z_{i+1}(\omega)\right)-f\left(z_{i}(\omega)\right)\right|<\varepsilon / 2\right\} .
\end{aligned}
$$

Because of the convergence mentioned above, we have

$$
P_{x_{0}}\left(\bigcup_{m=1}^{\infty} \bigcap_{i=m}^{\infty} A_{i}^{\varepsilon}\right)=1, \quad P_{x_{0}}\left(\bigcup_{m=1}^{\infty} \bigcap_{i=m}^{\infty} B_{i}^{\varepsilon}\right)=1,
$$

so by Lemma 3 and the strong Markov property we have

$$
\lim _{m \rightarrow \infty} P_{x_{0}}\left(\bigcap_{i=m}^{\infty} A_{i}^{\varepsilon} \mid y_{m}\right)=1, \quad \lim _{m \rightarrow \infty} P_{x_{0}}\left(\bigcap_{i=m}^{\infty} B_{i}^{\varepsilon} \mid z_{m}\right)=1 \quad P_{x_{0}} \text {-a.s., }
$$

and hence

$$
\lim _{m \rightarrow \infty} P_{x_{0}}\left(A_{m}^{\varepsilon} \mid y_{m}\right)=1, \quad \lim _{m \rightarrow \infty} P_{x_{0}}\left(B_{m}^{\varepsilon} \mid z_{m}\right)=1 \quad P_{x_{0}} \text {-a.s. }
$$

Now let $C^{\varepsilon}(x)=\{y \in D:|f(y)-f(x)|<\varepsilon / 2\}$. It is easy to see that

$$
P_{x_{0}}\left(A_{m}^{\varepsilon} \mid y_{m}\right)=P\left(y_{m}, C^{\varepsilon}\left(y_{m}\right)\right), \quad P_{x_{0}}\left(B_{m}^{\varepsilon} \mid z_{m}\right)=P\left(z_{m}, C^{\varepsilon}\left(z_{m}\right)\right)
$$

and so the right-hand sides converge to one almost surely. Let $\delta$ be as in Lemma 5 , and choose $m_{0} \geqq K$ so large that $m \geqq m_{0} \Rightarrow p\left(y_{m}, C^{\varepsilon}\left(y_{m}\right)\right) \geqq 1-\delta$. Choose $n_{0}(\omega)$ so large that $n \geqq n_{0} \Rightarrow$

$$
z_{n} \in \bigcup_{i=m_{0}}^{\infty} B\left(y_{m}, r\left(y_{m}\right)\right), \quad p\left(z_{n}, C^{\varepsilon}\left(t_{n}\right)\right) \geqq 1-\delta, \quad n_{0} \geqq K .
$$

Then let $m_{1} \geqq m_{0}$ satisfy $z_{n_{0}} \in B\left(y_{m_{1}}, r\left(y_{m_{1}}\right)\right)$. Applying Lemma 5 to the points $x_{1}=y_{m}, x_{2}=z_{n_{0}}, A_{1}=C^{\varepsilon}\left(y_{m}\right), A_{2}=C^{\varepsilon}\left(z_{n_{0}}\right)$ we see that $C^{\varepsilon}\left(y_{m}\right) \cap$ $C^{\varepsilon}\left(z_{n_{0}}\right) \neq \varnothing$, so there is an $x \in D$ with

$$
\left|f(x)-f\left(y_{m}\right)\right|<\varepsilon / 2 \text { and }\left|f(x)-f\left(z_{n_{0}}\right)\right|<\varepsilon / 2
$$

and thus $\left|f\left(y_{m}\right)=f\left(z_{n_{0}}\right)\right|<\varepsilon$. Because $K$ was arbitrary, we must have

$$
\left|\lim _{n \rightarrow \infty} f\left(y_{n}\right)-\lim _{n \rightarrow \infty} f\left(z_{n}\right)\right| \leqq \varepsilon
$$

whenever these limits exist, $P_{x_{0}}$-a.s. Hence $F=F \cdot \theta_{\eta} P_{x_{0}}$-a.s. and the proof is completed.

Proof of Theorem 2. Theorem 2 is proved in the same manner as Theorem 1, using instead the two-step transition probability for the 
Markov chains $y_{n}^{\prime}$ and $z_{n}^{\prime}$ given by

$$
y_{n}^{\prime}(\omega)=x\left(\sigma_{n}(\omega), \omega\right), \quad z_{n}^{\prime}(\omega)=y_{\eta}^{\prime}\left(\theta_{n}(\omega)\right) .
$$

4. Examples. The first example shows that some restrictions on $r$ are necessary. Let $D$ be the unit disc in $\boldsymbol{R}^{2}$, i.e., $\left\{(x, y): x^{2}+y^{2}<1\right\}$ and define

$$
\begin{array}{rlrl}
f(x, y) & =1, & & \text { if } y>0, \\
& =0, & & \text { if } y=0, \\
& =-1, & \text { if } y<0 .
\end{array}
$$

Let $r(x, y)=\min (|y|, 1-\|(x, y)\|)$ for $y \neq 0$ and $1-|x|$ for $y=0$. Then $f$ satisfies (1.1), is bounded and measurable, but not harmonic.

The second example shows that some restrictions on the growth of $f$ are necessary. Let $D=(0,1)$ in $R^{1}$ and define

$$
f(x)=\sum_{n=4}^{\infty} \varphi(n) 1_{[(n-1) / n, n /(n+1))}(x)
$$

where $\varphi(k)=0$, if $k=1,2,3$, and $\varphi(k)=(-1)^{k} k^{2}(k+1)$, for $k>3$.

Notice that

$$
\int_{0}^{n /(n+1)} f(x) d x=\sum_{k=4}^{n}(-1)^{k} k^{2}(k+1) \frac{1}{k(k+1)}=\sum_{k=4}^{n}(-1)^{k} k .
$$

Then if $g(x)=\int_{0}^{x} f(y) d y, \lim \sup _{x \rightarrow 1} g(x)=+\infty$ and $\lim \inf _{x \rightarrow 1} g(x)=$ $-\infty$ and $g$ is continuous on $[0,1)$. Moreover for any $x \in(1 / 2,1)$ the function

$$
h_{x}(r)=\frac{g(x+r)-g(x-r)}{2 r} \text { for } 0<r<1-x
$$

is continuous and also has lim sup and $\lim$ inf of $+\infty$ and $-\infty$ respectively as $r \rightarrow 1-x$. Thus there are values of $r$ arbitrarily near $1-x$ for which

$$
f(x)=h_{x}(r) \text {. }
$$

Clearly $r$ can be chosen a measurable function of $x$ to satisfy (4.1). For $0<x \leqq \frac{1}{2}$ one can define $r(x) \equiv \frac{1}{4}$. Thus $f$ and $r$ satisfy all of the hypotheses of our theorem except that of boundedness, and $f$ is not harmonic. (A similar example can be constructed in which $f$ is bounded and $D=(-\infty, \infty)$ by setting

$$
f(x)=\sum_{n=1}^{\infty}(-1)^{n}\left(1-\frac{1}{n}\right) 1_{\left[a_{n}, a_{n+1}\right)}(|x|)
$$

where $\left(a_{n}\right)$ increases sufficiently rapidly.) 
Finally, we remark that any bounded measurable periodic function on $\boldsymbol{R}^{1}$ satisfies the hypotheses for Theorem 2 with $r(x)$ equal to the period except that $N=1$. For further examples related to these concepts, see Courant and Hilbert [4].

\section{REFERENCES}

1. M. A. Ackoglu and R. W. Sharpe, Ergodic theory and boundaries, Trans. Amer. Math. Soc. 132 (1968), 447-460. MR 37 \#369.

2. J. R. Baxter, Restricted mean values and harmonic functions, Trans. Amer. Math. Soc. 167 (1972), 451-463. MR 45 \#2191.

3. K. L. Chung, The general theory of Markov processes according to Doblin, Z. Wahrscheinlichkeitstheorie und Verw. Gebiete 6 (1966), 203-223.

4. R. Courant and D. Hilbert, Methods of mathematical physics. Vol. II: Partial differential equations, Interscience, New York, 1962. MR 25 \#4216.

5. E. B. Dynkin, Markov processes. Vol. I, Fizmatgiz, Moscow, 1963; English transl., Die Grundlehren der math. Wissenschaften, Band 121, Academic Press, New York; Springer-Verlag, Berlin, 1965. MR 33 \#1886.

6. H. Föllmer, Ein Littlewood-Kriterium für gleichmässig integrable Martingale und insbesondere für Dirichlet-Lösungen, Elliptische Differentialgleichungen, Band II, Akademie-Verlag, Berlin, 1971.

7. W. A. Veech, The core of a measurable set and a problem in potential theory (preprint).

8. —_ A converse to Gauss' theorem, Bull. Amer. Math. Soc. 78 (1972), 444-446.

9. - - A zero-one law for a class of random walks and a converse to Gauss' mean value theorem (preprint).

Department of Mathematics, University of Minnesota, Minneapolis, MinneSOTA 55455

Current address: Université de Strasbourg, 67000 Strasbourg, France 